\title{
Levantamento Taxonômico e Sazonalidade de Calliphoridae, Muscidae e Fanniidae (Insecta: Diptera) em Feira de Santana, Bahia, Brasil
}

\author{
Thayana Teles Monteiro ${ }^{\bowtie}$, Evandro Nascimento da Silva \& Freddy Ruben Bravo
}

Universidade Estadual de Feira de Santana, e-mail: thayanamonteiro@yahoo.com.br (Autor para correspondência ${ }^{\bowtie}$ ), evandro.ecology@gmail.com, freddy11bravo@yahoo.com.br.

\section{EntomoBrasilis 7 (3): 171-177 (2014)}

Resumo. Os dípteros necrófagos utilizam matéria orgânica em decomposição como fonte proteica em ao menos uma fase de sua vida. Deste modo o conhecimento taxonômico dessa fauna se torna importante para estudos forense, sanitário e médico. Os dípteros das famílias Calliphoridae, Muscidae e Fanniidae possuem espécies necrófagas associadas ao ambiente humano, estando entre os insetos de maior destaque nesse aspecto e até então nunca foram estudados na cidade de Feira de Santana, Bahia. O objetivo deste estudo foi realizar um levantamento da fauna de dípteros necrófagos das famílias Calliphoridae, Muscidae e Fanniidae de Feira de Santana, Bahia. Para isso, foram realizadas coletas mensais ao longo de um ano em três áreas da cidade com um total de cinco armadilhas iscadas (com 24 horas de exposição) por área, resultando em um subconjunto de 60 armadilhas/ área e um total de 180 amostras. Foram coletados 4.818 dípteros, pertencentes a 20 espécies das três famílias estudadas: Calliphoridae (seis espécies), Fanniidae (três espécies) e Muscidae (11 espécies). A maior abundância das famílias ocorreu no outono e a menor no verão. Observou-se que treze das vinte espécies coletadas, possuem interesse forense e sanitário descritos na literatura.

Palavras-chave: Abundância; dípteros; moscas; riqueza de espécies.

\section{Taxonomic Survey and Seasonality of Calliphoridae, Muscidae and Fanniidae (Insecta: Diptera) in} Feira de Santana, Bahia, Brazil

Abstract. Taxonomic studies in necrphagous Diptera is relevant because of its importance for forensic, health and medical purposes, since they feed in decaying organic matter as protein source, at least in one stage of their lives. Synantropic Diptera, such us some species of Calliphoridae, Muscidae and Fannidae had not been studied in Feira de Santana, BA. The aim of this study was to do an inventory of necrophagous Diptera belonging to the families cited above. Five baited traps (24 hours of exposure) were placed in three sites in the town, once a month, resulting in a subset of 60 traps per site and a total of 180 samples. A total of 4.818 specimens were collected, belonging to 20 species: Calliphoridae (six species), Fannidae (three species) and Muscidae (11 species). The abundance of synantropic Diptera showed seasonality being the fall the season with higher abundance while in the summer the abundance was significant lower. Thirteen out of the twenty species collected were cited in the literature as being of forensic importance.

Keywords: Abundance; dipterans; flies; species richness.

$\sqrt{1}$ o Brasil, há um crescente interesse nos estudos dos dípteros muscóides, principalmente pelas famílias crófagas (Oliveira-Costa 2008; Amat 2010) Ess interesse consiste do fato de que ao ovi/larvipositarem em material orgânico animal em decomposição esses dípteros passam a ter uma importância na vigilância sanitária e saúde pública por atuarem como vetores mecânicos de vírus, bactérias, cistos de protozoários e ovos de helmintos (MARILUIs et al. 1989). Tal importância também pode ser observada para a entomologia forense por muitas serem necrófagas e atuarem na decomposição de corpos humanos (AMENDT et al. 2004; Oliveira-Costa 2008).

Inventários anteriores com dípteros muscóides necrófagos concentraram-se nos estados do Sul e Sudeste, onde a sinantropia desses dípteros é considerada bem estabelecida (FERREIRA 1978; LiNhARES 1981a, 1981b; D'Almeida 1992). Nos outros estados brasileiros os dados são insuficientes para alguns e atéinexistentes para outros (PuJol-Luz et al. 2008).

Na região Nordeste, a produção científica com dípteros necrófagos apresenta número ainda incipiente de publicações (e.g. ANDRADE et al. 2005; Oliveira \& Vasconcelos 2010; Vasconcelos \& Araujo 2012). Para a Bahia, existe apenas um trabalho, com um relato da fauna cadavérica na primeira década do século XX (FREIRE
1914).

A flutuação e composição das comunidades dos dípteros, como em outros insetos, estão diretamente ligadas a fatores bióticos e abióticos associados com o ecossistema em que eles se encontram (Mello et al. 2007; Gomes 2010). Portanto, é considerado de grande importância a realização de levantamentos por localidade com associações entre os fatores abióticos limitantes, visto que esses se alteram de região para região (OliveIra-Costa 2008).

Considerando a falta de estudos da dipterofauna necrófaga na região Nordeste, objetiva-se aqui contribuir com o conhecimento das espécies de dípteros das famílias Calliphoridae, Fanniidae e Muscidae ocorrentes em material orgânico animal e verificar a influência da sazonalidade sobre a abundância dessas espécies a partir de fatores abióticos na cidade de Feira de Santana, Bahia.

\section{MATERIAL E MÉTODOS}

O estudo foi realizado em três áreas arborizadas da cidade de Feira de Santana, estado da Bahia, localizadas nas regiões norte (Universidade Estadual de Feira de Santana, UEFS, $12^{\circ} 11^{\prime} 58,72$ "

Agência(s) de Fomento: CNPq (133864/2010-3) 
T S; $38^{\circ} 58^{\prime} 16,12 "$ O), centro-leste (Horto Florestal UEFS, $12^{\circ} 16{ }^{\prime} 12,07$ " S; $38^{\circ} 56$ '14,30" O) e sudeste (Parque da cidade Frei José Monteiro Sobrinho, $12^{\circ} 18^{\prime} 25,14^{\prime \prime} \mathrm{S} ; 38^{\circ} 56^{\prime} 29,82^{\prime \prime}$ O) do referido município. Durante um ano, entre maio de 2010 a abril de 2011, totalizaram-se 12 coletas, cada uma com periodicidade mensal. .

Em cada área colocou-se cinco armadilhas iscadas com 50 gramas de sardinha e moela de frango, modificadas de acordo com FerReira (1978) e Khouri (1995), com 24 horas de exposição ambiental prévia. As armadilhas foram deixadas no local por 24 horas. Após esse período, as moscas eram coletadas, levadas ao laboratório e mortas por resfriamento a cerca de $-10^{\circ} \mathrm{C}$ por pelo menos 10 minutos.

Os espécimes foram então identificados até nível de família e as que são objeto de estudo identificadas especificamente usando as seguintes chaves de identificação: AlbuQUERQue et al. (1981); Carvalho (2002); Carvalho et al. (2002); Mello (2003); Wendt \& CARvalho (2009). Os insetos identificados encontram-se depositados na Coleção Entomológica Prof. Johann Becker do Museu de Zoologia da Universidade Estadual de Feira de Santana (MZUEFS) e alguns exemplares da família Sarcophagidae, Muscidae e Fanniidae no Museu Nacional do Rio de Janeiro (MNRJ) servindo como material testemunho e coleção de referência para as famílias estudadas.

Realizou-se uma análise de correlação de Pearson através do programa Biostat 5.0 (AYres et al. 2007), entre a abundância absoluta de cada espécie e os valores das variáveis climáticas temperatura, pluviosidade e umidade relativa, cujos valores foram obtidos a partir do banco de dados temporário do INMET (20102011), sendo transformados em Log $\mathrm{x}$ antes da análise estatística. Para estimar a riqueza de espécies, utilizou-se estimadores de riqueza não-paramétricos através do programa EstimateS $\AA$
7.0 (Colwell 2004) a fim de obter as curvas de acumulação de espécies.

\section{RESULTADOS E DISCUSSÃO}

Coletou-se no total 4.818 indivíduos, distribuídos em 20 espécies (Tabela 1): três de Fanniidae - Fannia bahiensis Albuquerque, Fannia heydenii (Wiedemann) e Fannia pusio (Wiedemann); onze de Muscidae-Atherigona orientalis(Schiner), Cariocamyia maculosa Snyder, Cyrtoneurina costalis (Walker), Cyrtoneurina sp. nov., Cyrtoneuropsis dubia (Snyder), Cyrtoneuropsis polystigma (Wulpp), Graphomya analis (Macquart), Musca domestica (Linnaeus), Ophyra aenescens (Wiedemann), Ophyra chalcogaster (Wiedemann) e Synthesiomyia nudiseta (Wulp) e seis de Calliphoridae Chrysomya albicepis (Wiedemann), Chrysomya megacephala (Wiedemann), Chrysomya putoria (Wiedemann), Cochliomyia macellaria (Fabricius), Hemilucilia semidiaphana (Rondani) e Lucilia eximia (Widemann).

A riqueza de espécies estimada obtidas pelos estimadores de riqueza não-paramétricos (ACE, ICE, Chao 1 e 2 e Jackknife 1 e 2) (Figura 1). Os estimadores analisados demonstraram que no nosso estudo houve um esforço amostral adequado, pois a riqueza observada $\left(\mathrm{S}_{\mathrm{obs}}=20\right)$ variou entre $97,5 \%$ da riqueza real estimada por Chao 1 e 2 (menor estimativa, $S=20,5$ ) e $87 \%$ da riqueza real estimada pelo ACE (maior estimativa, $\mathrm{S}=23$ ). Segundo ReIS \& CANCELLO (2007). quando a riqueza observada atinge $85 \%$ da riqueza estimada, pode-se considerar que o esforço amostral foi satisfatório. Também corrobora esta análise o fato de que as curvas obtidas chegaram muito perto da estabilidade (assíntota) (Figura 1), demonstrando que o esforço amostral foi satisfatório e que a metodologia se mostrou eficaz. A partir da amostra 20, percebeu-se que mais de 80\% das espécies já tinham sido amostradas. Os estimadores Jackknife 2 e ACE estimaram os valores mais altos (22,98 e 23 espécies respectivamente). O

Tabela 1. Valores de abundância absoluta (n) e relativa (\%) observada para as espécies de dípteros coletados em Feira de Santana, Bahia, por estação do ano no período de maio de 2010 a abril de 2011.

\begin{tabular}{|c|c|c|c|c|c|c|c|c|c|c|}
\hline \multirow{2}{*}{ Espécies } & \multicolumn{2}{|c|}{ Inverno } & \multicolumn{2}{|c|}{ Primavera } & \multicolumn{2}{|c|}{ Verão } & \multicolumn{2}{|c|}{ Outono } & \multicolumn{2}{|c|}{ Total } \\
\hline & $\mathbf{n}$ & $\%$ & $\mathbf{n}$ & $\%$ & $\mathbf{n}$ & $\%$ & $\mathbf{N}$ & $\%$ & $\mathbf{N}$ & $\%$ \\
\hline Atherigona orientalis (Schiner) & 41 & 4,02 & 77 & 6,29 & 262 & 39,46 & 516 & 27,05 & 896 & 18,60 \\
\hline Cariocamya maculosa Snyder & 25 & 2,45 & 21 & 1,71 & o & o & 2 & 0,10 & 48 & 1,00 \\
\hline Chrysomya albicepis (Wiedemann) & 23 & 2,25 & 167 & 13,63 & 85 & 12,80 & 208 & 10,90 & 483 & 10,02 \\
\hline Chrysomya megacephala (Wiedemann) & 5 & 0,49 & 51 & 4,16 & 10 & 1,50 & 94 & 4,93 & 160 & 3,32 \\
\hline Chrysomya putoria (Wiedemann) & 2 & 0,20 & 4 & 0,33 & 1 & 0,15 & 52 & 2,73 & 59 & 1,22 \\
\hline Cochliomyia macellaria (Fabricius) & 2 & 0,20 & 9 & 0,73 & 3 & 0,45 & 24 & 1,26 & 38 & 0,79 \\
\hline Cyrtoneurina costalis (Walker) & 5 & 0,49 & 6 & 0,50 & o & o & 5 & 0,26 & 16 & 0,33 \\
\hline Cyrtoneurina sp. nov. & 12 & 1,17 & 1 & 0,08 & o & o & 5 & 0,26 & 18 & 0,37 \\
\hline Cyrtoneuropsis dubia (Snyder) & o & o & 1 & 0,08 & o & o & o & o & 1 & 0,02 \\
\hline Cyrtoneuropsis polystigma (Wulpp) & o & o & o & o & o & o & 1 & 0,05 & 1 & 0,02 \\
\hline Fannia bahiensis Albuquerque & 237 & 23,21 & 267 & 21,80 & 76 & 11,46 & 129 & 6,76 & 709 & 14,72 \\
\hline Fannia heydenii (Wiedemann) & 119 & 11,66 & 87 & 7,10 & 71 & 10,70 & 84 & 4,40 & 361 & 7,50 \\
\hline Fannia pusio (Wiedemann) & 406 & 39,76 & 359 & 29,30 & 89 & 13,40 & 534 & 28 & 1.388 & 28,81 \\
\hline Graphomya analis (Macquart) & o & o & o & o & 1 & 0,15 & o & o & 1 & 0,02 \\
\hline Hemilucilia semidiaphana (Rondani) & 4 & 0,39 & o & o & 2 & 0,30 & 21 & 1,10 & 27 & 0,56 \\
\hline Lucilia eximia (Widemann) & 58 & 5,68 & 33 & 2,70 & 4 & 0,60 & 67 & 3,51 & 162 & 3,36 \\
\hline Musca domestica (Linnaeus) & 6 & 0,59 & 22 & 1,80 & 34 & 5,12 & 121 & 6,34 & 183 & 3,80 \\
\hline Ophyra aenescens (Wiedemann) & 5 & 0,49 & 27 & 2,20 & o & o & 0 & 0 & 32 & 0,66 \\
\hline Ophyra chalcogaster (Wiedemann) & 11 & 1,08 & 26 & 2,12 & 2 & 0,30 & 4 & 0,20 & 43 & 0,89 \\
\hline Synthesiomyia nudiseta (Wulp) & 60 & 5,88 & 67 & 5,47 & 24 & 3,61 & 41 & 2,15 & 192 & 3,99 \\
\hline Total & 1.021 & 100 & 1.225 & 100 & 664 & 100 & 1.908 & 100 & 4.818 & 100 \\
\hline
\end{tabular}


estimador Jackknife 2 se baseia na incidência de espécies raras que ocorrem em apenas uma amostra (Singletons) bem como as que ocorrem em duas amostras (Doubletons) reduzindo o viés em duas vezes (DIAS 2004). Já o estimador ACE baseia-se na baixa abundância de espécies compartilhadas entre as amostras, ou seja, espécies com menos de 10 indivíduos por amostra (FERRAZ et al. 2009), esse fato foi comum no estudo, demonstrando assim um valor mais alto para o ACE (Figura 1).

Os estimadores Chao1 e 2 estimaram o valor mais próximo ao $\mathrm{S}_{\mathrm{obs}}(20,5)$. Chao 1 baseia-se na abundância das espécies raras de uma amostra enquanto que Chao 2 baseia-se na distribuição das espécies raras entre as amostras (COLWELL \& CODDINGTON 1994). Deste modo, esses estimadores foram os que melhor estimaram a fauna observada constatando que a região foi bem amostrada alcançando uma riqueza próxima da riqueza real.

O outono foi a estação que apresentou maior abundância entre as estações do ano (1.908 espécimes coletados) com valor médio de temperatura igual a $26^{\circ} \mathrm{C}$, pluviosidade total de $207 \mathrm{~mm}$ (com média de $47 \mathrm{~mm}$ nos meses de março e maio e $112 \mathrm{~mm}$ no mês de abril) e umidade média de $78 \%$. Já o verão obteve o menor valor de abundância (664 espécimes), com temperatura média igual a $27^{\circ} \mathrm{C}$, pluviosidade total de $152 \mathrm{~mm}$ (com o,8 mm para o mês de fevereiro) e umidade média igual a 77,4 (Figura 2). Essas estações foram as que demonstraram valores abióticos mais extremos, verificando uma relação também com a abundância.

Em trabalho de sazonalidade de insetos na caatinga nordestina, realizado por VASCONCELOS et al. (2010) os dípteros foram igualmente mais abundantes nas estações do outono e do inverno.

Esse fato indica uma maior ocorrência desses organismos nas estações mais frias, estando, provavelmente, os dípteros mais associados à ausência de chuvas e a temperaturas amenas.

Dentre as 20 espécies de dípteros muscóides coletados na cidade de Feira de Santana, Bahia, 13 possuem análise de sinantropia descrita em literatura: A. orientalis, C. albicepis, $C$. megacephala, C. putoria, C. macellaria, C. polystigma, F. pusio, $H$. semidiaphana, $L$. eximia, $M$. domestica, $O$. aenescens, $O$. chalcogaster e $S$. nudiseta.

Todas as espécies de Calliphoridae apresentaram valores de sinantropia acima de +25 , o que indica uma preferência por ambientes antropizados (LINHARES 1981a), sendo C. megacephala a espécie com maior grau de sinantropia (LINHARES 1981a; VIANNA et al. 1998), dentre as espécies aqui coletadas. Apenas $H$. semidiaphana não é considerada eussinantrópica (VIANNA et al. 1998).

Todos os valores de sinantropia calculados nos trabalhos analisados utilizam o índice proposto por NoRTUEva (1963) que atribui valores de -100 a +100 , em que os valores mais baixos representam espécies com aversão a áreas habitadas (assinantrópicas) e os valores mais altos incluem as espécies com alto grau de sinantropia.

Dentre os faniídeos coletados, apenas $F$. pusio foi encontrada em trabalho de sinantropia e demonstrou-se eussinantrópica com índice de +58 (LiNHARES 1981b).

Dos seis muscídeos ocorrentes em trabalhos de sinantropia, D'Almeida (1992) determinou $S$. nudiseta, A. orientalis e $M$. domestica como espécies altamente sinantrópicas com índices acima de +50 . LinHAREs (1981b) encontrou também $O$. chalcogaster e $O$. aenescens como espécies sinantrópicas, (+42). Das seis espécies acima citadas, todas podem ser consideradas sinantrópicas, visto sua abundância considerável encontrada neste trabalho (Tabela 1).

Para C. polystigma, Linhares (1981b) encontrou um valor de sinantropia de -100 , demonstrando que essa espécie possui aversão por áreas habitadas, sendo altamente assinantrópica, o que pode explicar a presença de apenas um exemplar nesse estudo, e mesmo assim, ocorrendo em área com a maior cobertura vegetal das áreas amostradas.

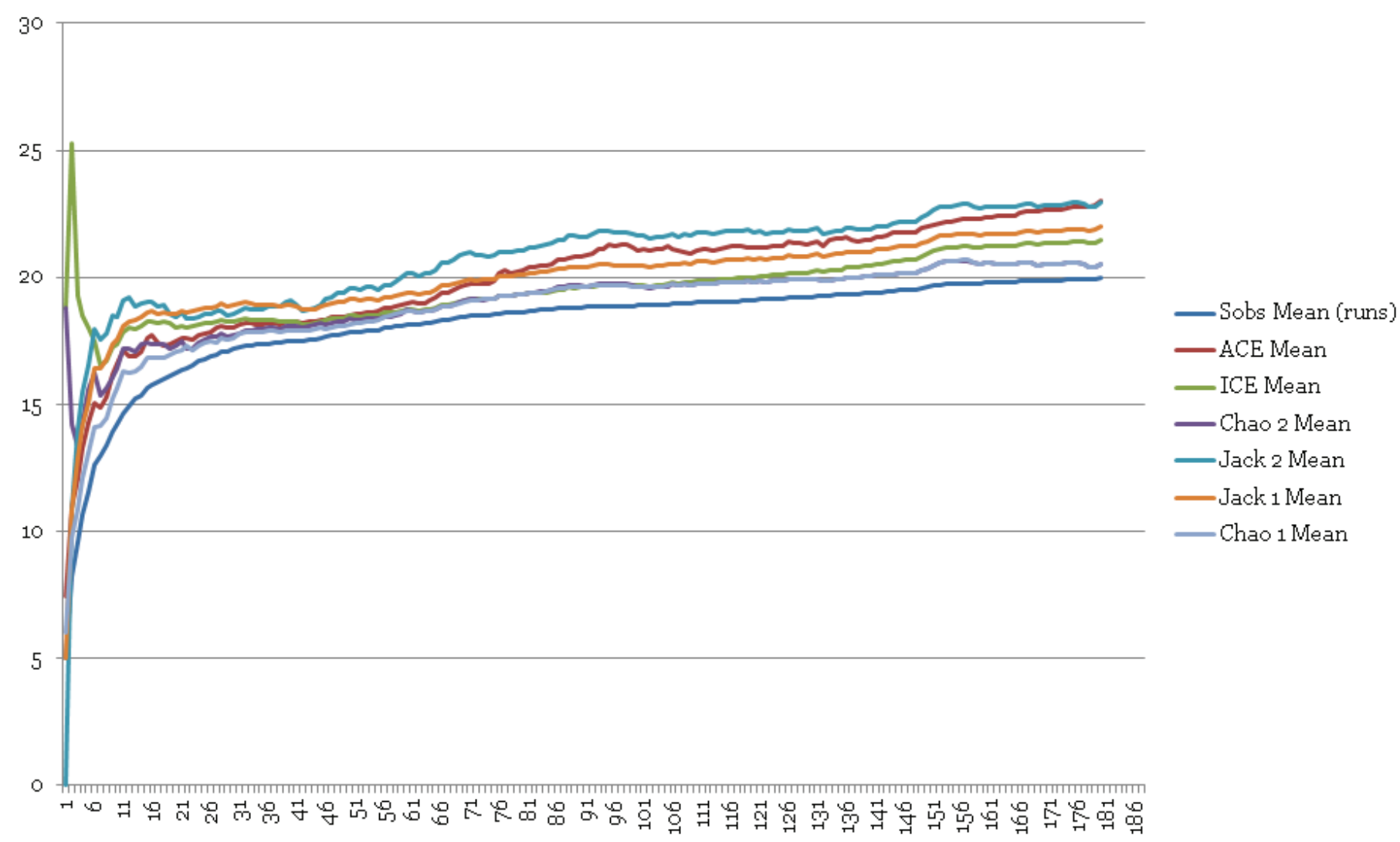

Figura 1. Gráfico com estimadores de riqueza não-paramétricos para amostras independentes (armadilhas) de dípteros coletados com iscas de origem animal em putrefação no período de maio de 2010 a abril de 2011 em Feira de Santana, Bahia. (S observado, ACE, ICE, Chao 1 e 2, Jackniffe 1 e 2). 
T

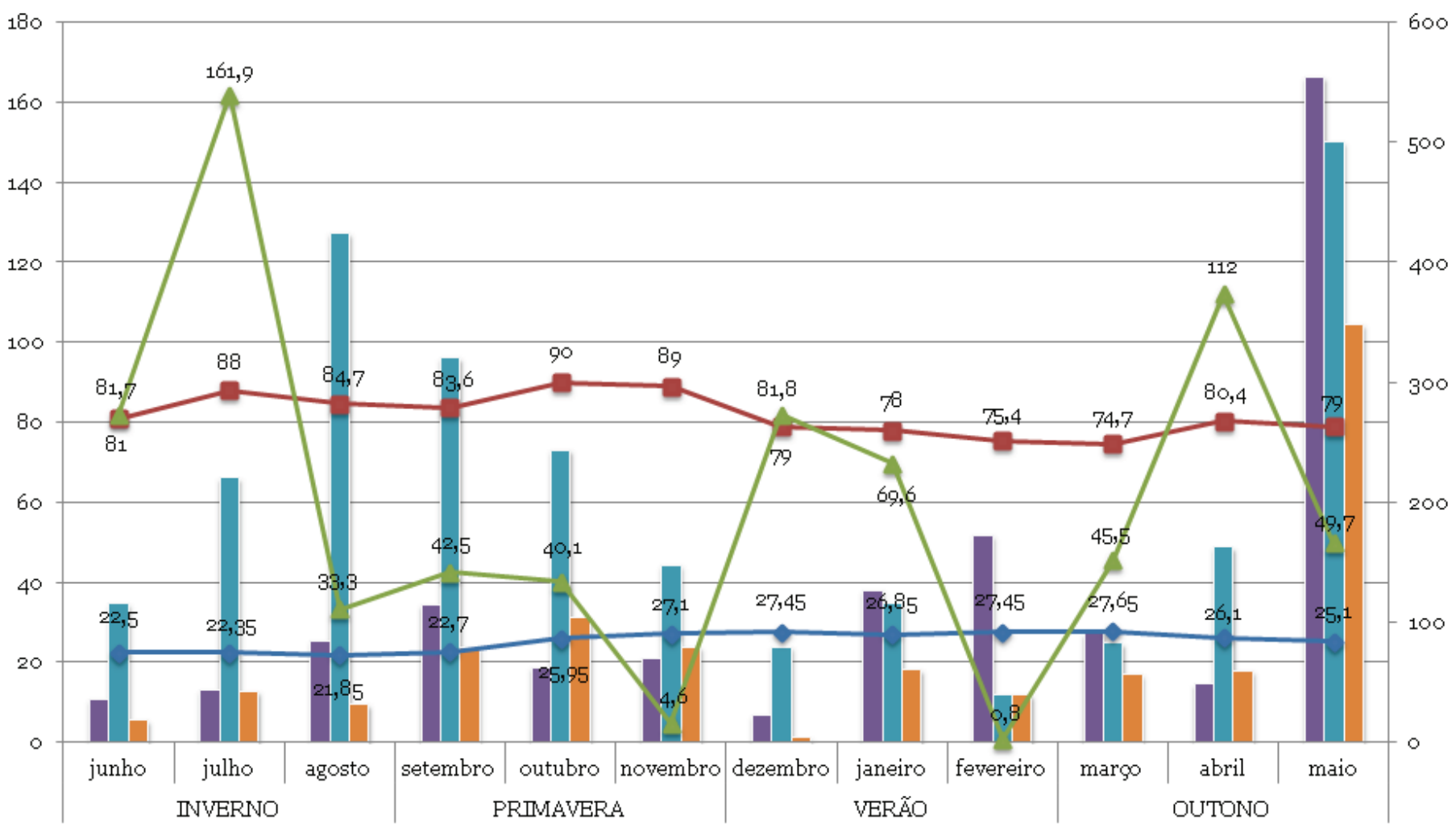

Wuscidae $=$ Fanniidae CCalliphoridae $\sim$ Temperatura Média $\left({ }^{\circ} \mathrm{C}\right)-$-Umidaderelativa (\%) -Pluviosidade(mm)

Figura 2. Abundância mensal de Calliphoridae, Muscidae e Fanniidae (colunas - eixo secundário) e variações dos fatores abióticos: umidade (\%), pluviosidade $(\mathrm{mm})$ e temperatura $\left({ }^{\circ} \mathrm{C}\right)$ (linhas - eixo primário), coletados com iscas de origem animal em putrefação no período de maio de 2010 a abril de 2011 em Feira de Santana, Bahia.

A família mais abundante das três coletadas foi Fanniidae, totalizando 2.458 indivíduos coletados, com pouco mais da metade do total $(51,02 \%)$. Este dado contrasta com trabalhos de levantamento em que Fanniidae apresenta-se como a menos abundante, como em LinHAREs (1981b) que coletou com iscas orgânicas em 10 coletas mensais ao longo de um ano, apenas 3,4\% de espécimes de Faniidae e LeAndro \& D'Almeida (2005) com $12,31 \%$ de fanniídeos dentre os espécimes coletados. Fanniidae obteve seu pico de abundância no inverno diferindo das demais famílias, que apresentaram pico no outono (Figura 2).

Apesar do elevado número de espécimes, Fanniidae apresentou a mais baixa riqueza, com apenas três espécies identificadas, $F$. bahiensis, $F$. heydenii e $F$. pusio, constantes em todos os meses de coleta. Dessas, $F$. pusio foi a mais representativa (total de 1.388 indivíduos), com maior abundância em cada uma das estações (Tabela 1) e demonstrando correlação negativa com a temperatura $(\mathrm{r}=-0,587 ; \mathrm{p}=0,0046)$, fato que explica sua maior abundância no inverno e outono. $F$. pusio possui importância forense estabelecida na literatura (Oliveira-Costa 2008) e tem distribuição ocorrente em praticamente todo o Brasil (CARVALHO et al. 2002).

A espécie $F$. bahiensis foi a terceira mais abundante (709 espécimes), entre os dípteros coletados, ocorrendo em maior quantidade no inverno e primavera (Tabela 1), o que corrobora com a correlação calculada, a qual é, negativa em relação à temperatura $(\mathrm{r}=-0,6149$ e $\mathrm{p}=0,0333)$ e positiva em relação à umidade $(\mathrm{r}=0,8346$ e $\mathrm{p}=0,0007)$.

Já $F$. heydenii ocorreu com maior abundância no inverno e primavera demonstrando correlação positiva em relação à temperatura $(\mathrm{r}=0,5611$ e $\mathrm{p}=0,0561)$ e umidade $(\mathrm{r}=0,5647$ e $\mathrm{p}$ = 0,0557). Gomes et al. (2002) relatou $F$. heydenii como vetor de ovos de Dermatobia hominis (Linnaeus) (Oestridae), oestrídeo responsável por causar míase primária em animais e humanos GoMEs et al. (2002).
A família Muscidae teve a segunda maior abundância (1.431 indivíduos) e a maior riqueza entre as famílias inventariadas, com 11 espécies representando 55\% do total coletado. A família apresentou seu pico de abundância no outono e queda no inverno (Figura 2).

As espécies A. orientalis, S. nudiseta, M. domestica e $O$. aenescens apresentaram a respectiva ordem de abundância e foram constantes em praticamente todos os meses de coleta. Essas espécies são amplamente distribuídas no Brasil e geralmente constantes em todos os trabalhos com muscídeos necrófagos.

A espécie $O$. aenescens é sinantrópica com importância relacionada ao seu comportamento agressivo frente a $M$. domestica, agindo como reguladora de populações desse muscídeo (SKIDMORE 1985). Esse comportamento pode ser observado neste trabalho devido ao contraste de abundância entre as duas. No mês de maior abundância de $M$. domestica (79 espécimes), $O$. aenescens não esteve presente, e no mês em que esta foi mais abundante (27), apenas três espécimes de $M$. domestica foram identificadas, demonstrando um provável comportamento competitivo entre ambas.

O hábito saprófago e a importância econômica forense e sanitária de $A$. orientalis, $S$. nudiseta, $M$. domestica, $O$. aenescens e $O$. chalcogaster são dados bem estabelecidos na literatura (e.g., Rodríguez \& Leite 1997; Rodríguez et al. 1999; Oliveira-Costa 2008).

O muscídeo $C$. maculosa é pouco comum em trabalhos de levantamentos, tendo sua biologia ainda pouco conhecida, mas com tendência à necrofagia, conforme especulado por D'AlmEidA (1994) e Monteiro \& Bravo (2011), tendo sido registrada em iscas de sardinha e fígado de galinha, na caatinga nordestina (VASCONCELOS \& ARAÚJO 2012). Essa espécie foi mais frequente no inverno e primavera, sendo ausente no verão e com a coleta de apenas dois espécimes no outono. 
Dentre as demais espécies, C. dubia, C. polystigma e G. analis foram representadas por um único exemplar de cada um, respectivamente (Tabela 1 ). Tal fato pode estar relacionado a alguma ineficiência da metodologia ora empregada, sendo necessário estudos mais prolongados e direcionados, já que é sabido da ocorrência de alguns exemplares dessas espécies em substratos orgânicos, como relatado por Couri et al. (2000), que coletou C. dubia com sardinha e fezes no Amapá, entre 25\% dos Muscidae levantados, LiNHAREs (1981b), que coletou 55 espécimes de C. polystigma atraídas por fezes em Campinas, SP. Em seu estudo D'Almeida (1991), no Rio de Janeiro, coletou um exemplar de $G$. analis, utilizando peixe como isca, enquanto VASCONCELOS \& ARAúJo (2012), em área urbana de Recife-PE, registraram $G$. analis em iscas de sardinha e fígado de galinha (quantidade não informada).

Calliphoridae foi a família menos representativa em termos de abundância total, com apenas 929 indivíduos coletados. Essa amostragem contraria estudos semelhantes em que Calliphoridae apresentou a maior abundância (e.g. LEANDRo \& D'ALMEIDA 2005; FurusaWa \& CASSINO 2006; Amat 2010). A família apresentou pico de abundância no outono e primavera com baixa considerável no verão (Figura 2).

Dentre as espécies de Calliphoridae identificadas, as do gênero Chrysomya, foram as mais constantes e abundantes (Tabela 1), representando $75,56 \%$ dos califorídeos encontrados. O gênero Chrysomya inclui espécies do Velho Mundo introduzidas nas Américas na década de 70 e que rapidamente se dispersaram pelo continente a partir do sudeste Brasileiro, alcançando as áreas antropizadas, estabelecendo-se nos centros urbanos ou próximos a estes, tornando-se alvo da vigilância sanitária (GUIMARÃEs et al. 1979; BAUMGARTNER 1988).

Todas as espécies de Calliphoridae identificadas no presente trabalho são corriqueiras em estudos com matéria orgânica e possuem interesse forense descrito na literatura (e.g., FrEIRE 1914; Oliveira-Costa 2008; Carvalho \& Mello-Patiu 2008).

A espécie $C$. albicepis foi o califorídeo mais abundante (483 espécimes), presente em todas as estações do ano (Tabela 1). Essa alta ocorrência é um dado corroborado em trabalhos com metodologias semelhantes (e.g. Vianna et al. 1998; FerRaz et al. 2010). Segundo MARChiori et al. (2000) e BiavatTi et al. (2010), o hábito voraz e a competitividade larval de C. albicepis torna-a capaz de deslocar espécies nativas como $C$. macellaria e $H$. semidiaphana, fato que pode ter ocorrido neste estudo, devido a baixa abundância total encontrada nessas duas espécies: 1,22\% e 0,56\% respectivamente (Tabela1).

A baixa abundância observada para $H$. semidiaphana (27 espécimes - Tabela 1) pode ser explicada também pelo fato desta espécie ser assinantrópica comum (FERRAz et al. 2010), sendo mais corriqueira e abundante em estudos realizados em matas e florestas. Carvalho \& Linhares (2001) afirmou que $H$. semidiaphana é uma espécie indicadora de áreas florestadas, fato corroborado nesse trabalho pela maior abundância desta espécie na área 3 (19 exemplares), que possui predominância de porte arbóreo. Em contraste, encontrou-se apenas dois exemplares na área 2, predominantemente urbana e com poucas árvores.

A espécie L. eximia apresentou-se como a segunda Calliphoridae mais abundante (162 indivíduos - Tabela 1), tendo sua ocorrência mais associada ao outono e ao inverno, assim como observado por MELLo et al. (2007), o que pode ser explicado pela correlação negativa entre sua abundância e a temperatura $(r=-0,7468$ e $p$ $=0,0052$ )

A terceira espécie mais abundante, C. megacephala (160 espécimes - Tabela 1), apresentou boa constância em todas as estações, e sua abundância em trabalhos semelhantes no Brasil é predominante (e.g. FuruSAWA \& CASSINO 2006; BATISTA-DA-Silva et al. 2010; GONÇALVES et al. 2011).
Do gênero Chrysomya, C. putoria apresentou a menor abundância total (59 indivíduos - Tabela 1), representando 8,4\% de todas as Chrysomya coletadas. A sua baixa incidência reflete as conclusões de Souza \& LinHAREs (1997), que afirmaram que $C$. putoria não utiliza substrato putréfico para oviposição, mas sim para ganho proteico.

Levando-se em consideração que nenhum estudo sobre a fauna de interesse forense e de importância sanitária havia sido feito para a cidade de Feira de Santana até então, e que esse trabalho é pioneiro na região, os dados aqui levantados subsidiam os primeiros registros sobre a fauna de interesse econômico da cidade e região.

\section{AGRADECIMENTOS}

À professora Dr. Márcia Couri (MNRJ) pelas considerações e pelo auxílio na identificação das espécies. Ao CNPq pela concessão da bolsa de mestrado (133864/2010-3).

\section{REFERÊNCIAS}

Albuquerque, D.O., D. Pamplona \& C.J.B. Carvalho, 1981. Contribuição ao conhecimento dos Fannia R.D., 1830 da região Neotropical (Diptera, Fanniidae). Arquivos do Museu Nacional, 56: 9-34.

Amat, E., 2010. Notes on necrophagus flies (Diptera: Calyptratae) associated to fish carrion in Colombian Amazon, Acta Amazonica, 40: 397-400.

Amendt, J., R. Krettek \& R. Zehner, 2004. Forensic entomology. Naturwissenchaften, 91: 51-65.

Andrade, H.T.A., A.A. Varela-Freire, M.J.A. Batista \& F. Jansen, 2005. Calliphoridae (Diptera) coletados em cadáveres humanos no Rio Grande do Norte. Neotropical Entomology, 34: 855-856.

Ayres, M., M. Ayres Junior, D.L. Ayres \& A.A.S. Santos, 2007. BioEstat 5.0: aplicações estatísticas nas áreas das Ciências Biomédicas. Sociedade Civil Mamirauá: Belém, Pará-Brasil, 324p.

Batista-Da-Silva, J.A., G.E. Moya-Borja \& M.M.C. Queiroz, 2010. Ocorrência e sazonalidade de muscóides (Diptera, Calliphoridae) de importância sanitária no Município de Itaboraí, RJ, Brasil. EntomoBrasilis, 3: 16-21.

Baumgartner, D.L., 1988. Spread of introduced Chrysomya Blowflies (Diptera, Calliphoridae) on the Neotropics with records new to Venezuela. Biotropica, 20: 167-168.

Biavatti, G.M., F.H.A. Santana \& J.R. Pujol-Luz, 2010. A checklist of Calliphoridae Blowflies (Insecta, Diptera) associated with a pig carrion in Central Brazil. Journal of Forensic Sciences, 55: $1603-1605$.

Carvalho, C.J.B. \& C.A. Mello-Patiu, 2008. Key to the adults of the most common forensic species of Diptera in South America. Revista Brasileira de Entomologia, 52: 390-406.

Carvalho, C.J.B. (Editor), 2002. Muscidae (Diptera) of the Neotropical Region: Taxonomy. Editora UFPR. Curitiba, Paraná. 287p.

Carvalho, C.J.B., M.O. Moura \& P.B. Ribeiro, 2002. Chave para adultos de dípteros (Muscidae, Fanniidae, Anthomyiidae) associados ao ambiente humano no Brasil. Revista Brasileira de Entomologia, 46: 107-114.

Carvalho, L.M.L. \& A.X. Linhares, 2001. Seasonality of insects succession and pig carcass decomposition in a natural forest area in southearstern Brazil. Journal of Forensic Science, 46: 604-608.

Colwell, R.K. \& J.A. Coddington, 1994. Estimating terrestrial biodiversitythrough extrapolation.PhilosophicalTransactions of the Royal Society of London B, 345: 101-118.

Colwell, R.K., 2004. EstimateS, Version 7.0: Statistical Estimation of Species Richness and Shared Species from Samples (Software and User's Guide). Freeware for Windows and Mac OS. Disponível em: < http://viceroy.eeb.uconn.edu/ EstimateS/>. 
Couri, M.S., C.J.E. Lamas, C.C.de C. Aires, C.A. de Mello-Patiu, V.C. Maia, D.M. Pamplona \& P. Magno, 2000. Diptera da Serra do Navio (Amapá, Brasil): Asilidae, Bombyliidae, Calliphoridae, Micropezidae, Muscidae, Sarcophagidae, Stratiomyiidae, Syrphidae, Tabanidae e Tachinidae. Revista Brasileira de Zoociências, 2: 81-90

D’Almeida, J.M., 1991. Dípteros caliptrados (Muscidae e Anthomyiidae) da região metropolitana do Rio de Janeiro, RJ. II. Atratividade e freqüência sazonal. Revista Brasileira de Zoologia, 8: 7-16.

D’Almeida, J.M., 1992. Calyptrate Diptera (Muscidae and Anthomyidae) of the state of Rio de Janeiro - 1. Synanthropy. Memórias do Instituto Oswaldo Cruz, 87: 381-386.

D'Almeida, J.M., 1994. Ovipositional substrates used by Calyptrate Diptera in Tijuca Forest, Rio de Janeiro. Memórias do Instituto Oswaldo Cruz, 89: 261-264.

Dias, S.C., 2004. Planejando estudos de diversidade e riqueza: uma abordagem para estudantes de graduação. Acta Scientiarum Biological Sciences, 26: 373-379.

Ferraz, A.C.P., B.Q. Gadelha \& V.M. Aguiar-Coelho, 2009. Análise faunística de Calliphoridae (Diptera) da Reserva Biológica do Tinguá, Nova Iguaçu, Rio de Janeiro. Revista Brasileira de Entomologia, 53: 620-628.

Ferraz, A.C.P., B.Q. Gadelha \& V.M. Aguiar-Coelho, 2010. Influência climática e antrópica da abundância e riqueza de Calliphoridae (Diptera) em fragmento da Reserva Biológica do Tinguá, RJ. Neotropical Entomology, 39: 476-485.

Ferreira, M.J.M., 1978. Sinantropia de dípteros muscoídeos de Curitiba, Paraná. I. Calliphoridae. Revista Brasileira de Biologia, 38: 445-454.

Freire, O., 1914. Algumas notas para o estudo da fauna cadavérica na Bahia. Gazeta Médica da Bahia, XLVI: 149-162.

Furusawa, G.P. \& P.C.R Cassino, 2006. Ocorrência e distribuição de Calliphoridae (Diptera, Oestroidea) em um Fragmento de Mata Atlântica secundária no município de Engenheiro Paulo de Frontin, Médio Paraíba, RJ. Revista de Biologia e Ciências da Terra, 6: 152-164.

Gomes, L. (Org.), 2010. Entomologia forense: novas tendências e tecnologias nas Ciências Criminais. $1^{\text {a }}$ Edição. Rio de Janeiro, Technical Books, $240 \mathrm{p}$.

Gomes, P.R., W.W. Koller, A. Gomes; C.J.B. Carvalho \& R. Zorzatto, 2002. Dípteros fanídeos vetores de ovos de Dermatobia hominis em Campo Grande, Mato Grosso do Sul. Pesquisas Veterinárias Brasileiras, 22: 114-118.

Gonçalves, L., A. Dias, C.B. Espíndola \& F.S. Almeida, 2011. Inventário de Calliphoridae (Diptera) em Manguezal e fragmento de Mata Atlântica na região de Barra de Guaratiba, Rio de Janeiro, Brasil. Revista Brasileira de Biociências, 9: 50-55.

Guimarães, J.H., A.P. Prado \& G.M. Buralli, 1979. Dispersal and distribuition of three newly introduced species of Chrysomya Robineau-Desvoidy in Brazil (Diptera, Calliphoridae). Revista Brasileira de Entomologia, 23: 245-255.

Khouri, A., 1995. Notas sobre a confecção de uma rede de coleta e armadilha especialmente para Dípteros caliptratos em lixo. Boletim del Museu Entomológico de la Universidad del Valle, 3: $55-59$.

Leandro, M.J.F. \& J.M. D’Almeida, 2005. Levantamento de Calliphoridae, Fanniidae, Muscidae e Sarcophagidae em um fragmento de mata na Ilha do Governador, Rio de Janeiro, Brasil. Iheringia, Série Zoologia, 95: 377-381.

Linhares, A.X., 1981a. Synanthropy of Calliphoridae e Sarcophagidae (Diptera) in the city of Campinas, São Paulo, Brazil. Revista Brasileira de Entomologia, 25: 231-243.

Linhares, A.X., 1981b. Synanthropy of Muscidae, Fanniidae and Anthomyiidae (Diptera) in the city of Campinas, São Paulo, Brazil. Revista Brasileira de Entomologia 25: 231-243.

Marchiori, C.H., C.G Silva, E.R. Caldas, C.I.S. Vieira, K.G.S. Almeida, F.F. Teixeira \& A.X. Linhares, 2000. Artrópodes associados com carcaças de suíno em Itumbiara, sul de Goiás. Arquivos do Instituto Biológico, São Paulo, 67: 167-170.
Mariluis, J.C., M.C. Lagar \& E.J. Bellegarde, 1989. Disseminacion de enteroparasitos por Calliphoridae (Insecta, Diptera). Memórias do Instituto Oswaldo Cruz, 84: 349-351.

Mello, R.P., 2003. Chave para a identificação das formas adultas das espécies da família Calliphoridae (Diptera: Brachycera, Cyclorrhapha) encontradas no Brasil. Entomologíay Vectores, 10: 255-268.

Mello, R.S., M.M.C. Queiroz \& V.M. Aguiar-Coelho, 2007. Population fluctuations of calliphorid species (Diptera, Calliphoridae) in Biological Reserve of Tinguá, state of Rio de Janeiro, Brazil. Iheringia, Série Zoologia, 97: 481-485.

Monteiro, T.d'A. \& F. Bravo, 2011. Diptera, Muscidae, Cariocamyia maculosa Snyder: Primeiro Registro para o Nordeste do Brasil. EntomoBrasilis, 4: 154-156.

Nortueva, P., 1963. Synanthropy of blowflies (Diptera, Calliphoridae) in Finland. Annals of Entomology Fennici, 29: 1-49.

Oliveira, T.C. \& S.D. Vasconcelos, 2010. Insects (Diptera) associated with cadavers at the Institute of Legal Medicine in Pernambuco, Brazil: implications for forensic entomology. Forensic Science International, 198: 97-102.

Oliveira-Costa, J., 2008. Entomologia Forense: quando os insetos são vestígios. $2^{\mathrm{a}}$ edição. Série Tratados de Perícias Criminalísticas. São Paulo, Millenium, 456p.

Pujol-Luz, J.R., L.C. Arantes \& R. Constanino, 2008. Cem anos da entomologia forense do Brasil (1908-2008). Revista Brasileira de Entomologia, 52: 485-492.

Reis, Y.T. \& E. M. Cancello, 2007. Riqueza de cupins (Insecta, Isoptera) em áreas de Mata Atlântica primária e secundária do sudeste da Bahia. Iheringia: Série Zoologia, 97: 229-234.

Rodríguez, Z. \& R.C. Leite, 1997. Ocorrência de vetores biológicos da Dermatobia hominis (L.Jr., 1971) (Diptera: Cuterebridae), capturados com armadilha Magoon na região metalúrgica do estado de Minas Gerais, MG. Ciência Rural, 27: 645-649.

Rodríguez, Z., R.C. Leite \& P.R. Oliveira, 1999. Ophyra aenescens (L.) (Diptera: Muscidae) a New Biological Vector of Dermatobia hominis (L. Jr) (Diptera: Cuterebridae) in Minas Gerais, Brazil. Memórias do Instituto Oswaldo Cruz, 94: 53-54.

Skidmore, P., 1985. The biology of the Muscidae of the world. Junk, Dordrecht. Series entomologica, 29, xiv + 550p

Souza, A.M. \& A.X. Linhares, 1997. Diptera and Coleoptera of potencial forensic importance in southeastern Brazil: relative abundance and seasonality. Medical and Veterinary Entomology, 11: 8-12.

Vasconcellos A., R. Andreazze, A.M. Almeida, H.F.P. Araujo, E.S. Oliveira \& U. Oliveira, 2010. Seasonality of insects in the semi-arid Caatinga of northeastern Brazil. Revista Brasileira de Entomologia, 54: 471-476.

Vasconcelos, S.D. \& M.C.S. Araujo, 2012. Necrophagous species of Diptera and Coleoptera in northeastern Brazil: state of the art and challenges for the Forensic Entomologist. Revista Brasileira de Entomologia, 56: 7-14.

Vianna, E.E.S., J.G.W. Brum, P.B. Ribeiro, M.E.A. Berne \& P. Silveira-Junior, 1998. Synanthropy of Calliphoridae (Diptera) in Pelotas, Rio Grande do Sul state, Brazil. Revista Brasileira de Parasitologia Veterinária, 7: 141-147.

Wendt, L.D. \& C.J.B. Carvalho, 2009. Taxonomia de Fanniidae (Diptera) do sul do Brasil - II: Novas espécies e chave de identificação de Fannia Robineau-Desvoidy. Revista Brasileira de Entomologia 53: 171-206.

\section{Recebido em: 10/05/2013}

Aceito em: 01/11/2014 
Como citar este artigo:

Monteiro, T.T., E.N. Silva \& F.R. Bravo, 2014. Levantamento Taxonômico e Sazonalidade de Calliphoridae, Muscidae e Fanniidae (Insecta: Diptera) em Feira de Santana, Bahia, Brasil. EntomoBrasilis, 7 (3): 171-177.

Acessível em: doi:10.12741/ebrasilis.v7i3.352
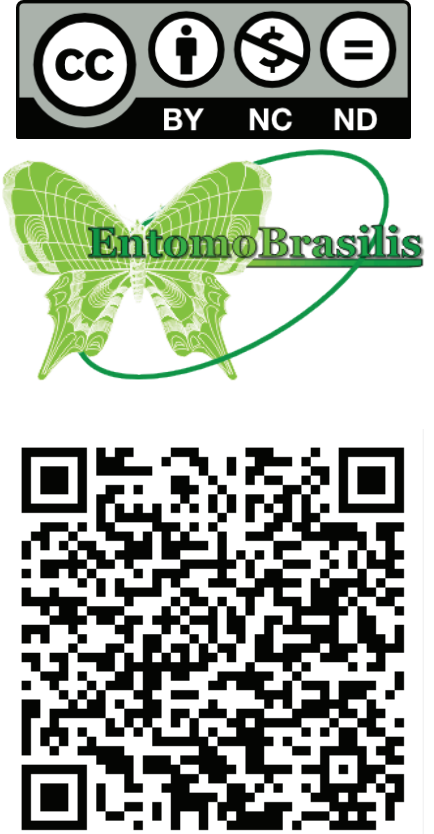